Supporting Information for:

\title{
Nanoscale Infrared Spectroscopy and Chemometrics Enable Detection of Intracellular Protein Distribution
}

\author{
A. Catarina V. D. dos Santos ${ }^{\dagger}$, Rosa Heydenreich ${ }^{\ddagger}$ Christian Derntl ${ }^{\ddagger}$, Astrid R. Mach-Aigner ${ }^{\ddagger}$, Robert \\ Mach ${ }^{\ddagger}$, Georg Ramer ${ }^{\dagger, *}$, Bernhard Lendl ${ }^{\dagger}$ \\ ${ }^{\dagger}$ Institute of Chemical Technologies and Analytics, TU Wien, 1060 Vienna, Austria \\ ${ }^{\ddagger}$ Institute of Chemical, Environmental and Bioscience Engineering, TU Wien, 1060 Vienna, Austria \\ *Corresponding author email: georg.ramer@tuwien.ac.at
}

\section{Table of contents}

Figure S1 Comparison of spectra for different T.Reesei strains 


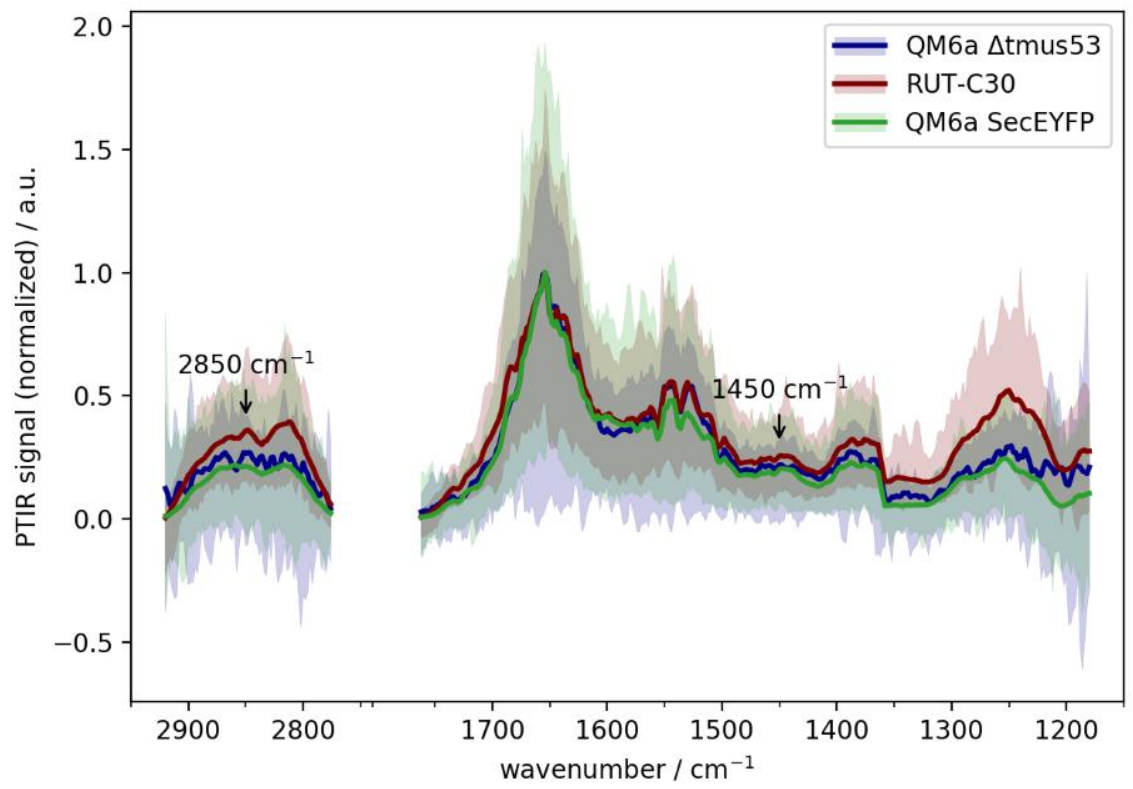

Figure S1: PTIR spectra of wild type (QM6a $\Delta t m u s 53$ ), strain used in manuscript measurements (QM6a SecEYFP) and a cellulase super producer strain (RUT-C30). The similarities between wild type and SecEYFP spectra, suggest that EYFP expression levels do not happen at a level at which PTIR spectra are affected. This is in accordance with literature. ${ }^{1}$ Furthermore, when comparing the RUTC30 strain to the SecEYFP, it is possible to see an increase of signal at both $2850 \mathrm{~cm}^{-1}$ and $1450 \mathrm{~cm}^{-1}$, which reinforces our hypothesis that these signals come from transport vesicles. Interestingly, the flank of the amide I band in the super producer spectrum also hints at an increase in beta sheet protein, consistent with this strain's characteristic cellulase super production. A major reconfiguration of the beam path between recording the spectra in the main matter and in this plot means that relative intensities in this plot cannot be compared directly with those in figure 1.

(1) Nyyssönen, E.; Keränen, S. Multiple Roles of the Cellulase CBHI in Enhancing Production of Fusion Antibodies by the Filamentous Fungus Trichoderma Reesei. Curr. Genet. 1995, 28 (1), 71-79. https://doi.org/10.1007/BF00311884. 